\title{
Forced Vibration Analysis Of Rectagular Plates Usinig Higher Order Finite Layer Method
}

\author{
A. A. Abdul-Razzak \\ J. H. Haido \\ Assistant Professor \\ Assistant Lecturer \\ Civil Engineering Department \\ Civil \\ Engineering Department \\ Mosul, Iraq \\ Mosul University, \\ Dohuk University, Dohuk, Iraq
}

\begin{abstract}
In this paper a higher order finite layer formulation based on the auxiliary nodal surface (ANS) technique for a forced vibration analysis of rectangular plates is presented. The forced vibration analysis has been performed using the Newmark integration method for investigating the vibration characteristics and finding the response of rectangular plates under the action of dynamic loads. The forced vibration of the rectangular plates subjected to moving and impact loads has been studied.

Several examples have been studied to show the good performance of the higher order finite layer with one ANS for forced vibration analysis of plate.
\end{abstract}

Keywords: Finite layer, Forced Vibration, Thick Plate. 
تحليل الاهتزاز القسري للصفائح المستطيلة باستخدام طريقة الطبقة المحدة ذات النسق العالي

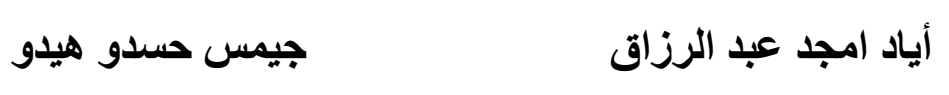

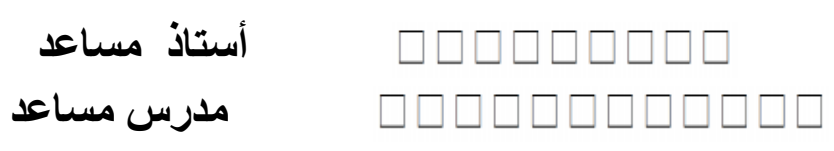

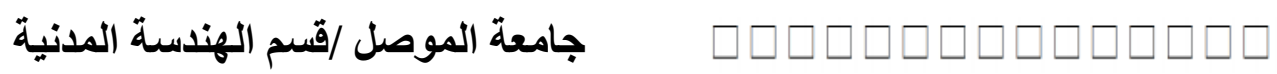

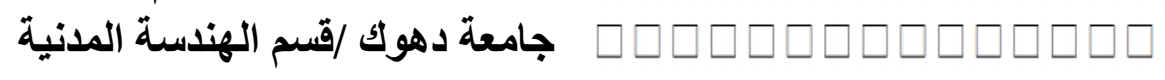

\section{الخلاصة}

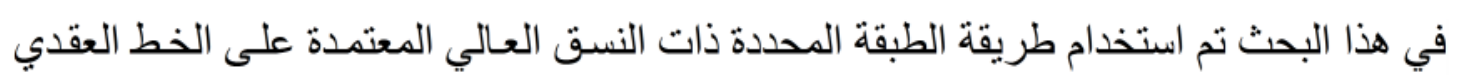

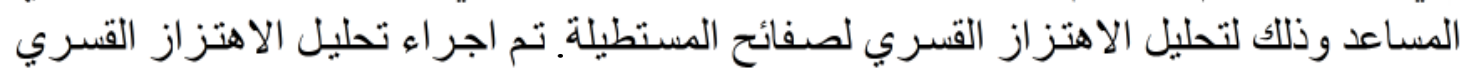

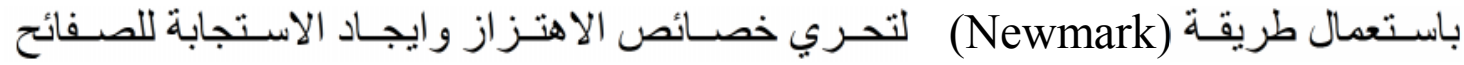

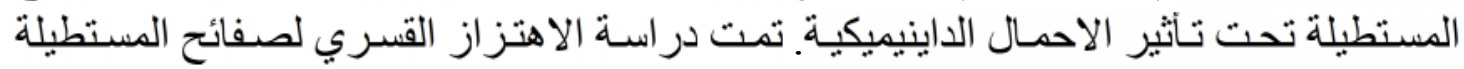

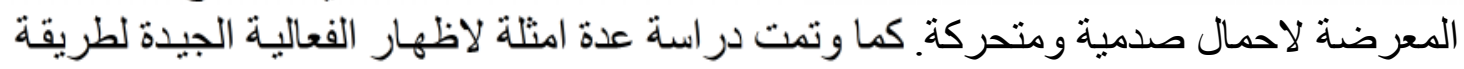

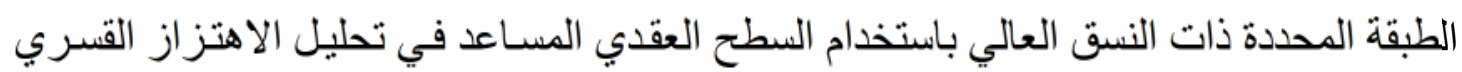

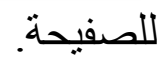

Received 19 July 2007

Accepted 10 Dec. 2007

\section{Notation:}

$\mathrm{a}, \mathrm{b} \quad$ Length and width of plate

[B] Strain matrix

[C] Coefficient matrix for displacement function

c Thickness of plate

[D] Elasticity matrix 
DAF Dynamic Amplification Factor

E Modulus of elasticity

$\{f\} \quad$ Displacement function

h Thickness of layer

$\mathrm{m}, \mathrm{n} \quad$ Particular harmonic number in $\mathrm{X}$ and $\mathrm{Y}$-direction

[M] Mass matrix

$\mathrm{r}, \mathrm{s} \quad$ Specified number of harmonic terms in $\mathrm{x}$ and $\mathrm{y}$-directions

[S] Stiffness matrix

$\{\ddot{\delta}\},\{\dot{\delta}\},\{\delta\}$ Vectors of nodal displacements, velocities and Accelerations

$\mathrm{x}, \mathrm{y} \quad$ Global coordinate in length -wise and width-wise direction

$\mathrm{Xm}, \mathrm{Yn}$ Harmonic function in $\mathrm{x}$ and $\mathrm{y}$-directions

$\mathrm{z} \quad$ Global coordinate in thickness -wise direction

$\{\phi\} \quad$ Eigen vector

$\rho \quad$ Mass per unit volume

$v \quad$ Poisson's ratio

$\omega \quad$ Natural frequency $(\mathrm{rad} / \mathrm{sec})$

\section{Introduction:}

Rectangular plates are the main parts in various structures as bridges, hydraulic, water tanks.... etc; in these structures the plate is an important component in carrying directly the applied loads.

The dynamic behavior of the structure is defined by a special frequency spectrum consisting of an infinite number of natural frequencies and mode shapes which can be found by knowing the 
geometrical shape, mass distribution, stiffness and boundary conditions of the plates [1].

It is necessary to understand the dynamic response of different type of rectangular plates such as isotropic plates, orthotropic plates, sandwich plates and laminated plates because of greater increase in the dynamic deformation compared to the static deformation.

Plates may be subject to dynamical effects in the form of loads, which lead to vibrate the plate. These loads may be moving forces on the surface of the plate, its location may be fixed on the plate but its magnitude or its direction is changing with the time such as the impact loads and explosions. There are many cases of the applied loads on plates as uniformly distributed loads, patch loads and concentrated loads. ...etc. It is of necessity to consider the dynamic effect of these loads on plates in the structural analysis and design because of preventing the effects of the applied loads to be accompanied with any damages which may lead to failure.

Many researchers have studied the effects of some parameters on the vibration of plates subjected to dynamic loads. In 1982 [2], analytical results are found for the dynamic interaction of an elastic flexural plate and an elastic half-space subjected to harmonic seismic waves. Displacements and contact stresses are found for square, massless plates having a particular range of flexural stiffness and subjected to incident waves oriented parallel to either an edge or a diagonal of the plate. It is found that the plate exhibits additional resonance.

At the beginning of nineties of the last century and especially in 1990 [3] an algorithm based on a finite element approach has been developed by Mechael and Ting to study the transient response of rectangular plates having arbitrary boundary conditions and subjected to a moving force. Thin plate theory is used for the plate model with no restriction is placed on the loading conditions. The algorithm accounts for the complete dynamic interactions between the moving loads and the plate. Therefore, the method can be applied to the general moving mass problems and also to the simplified moving force and static problems. The dynamic response of isotropic rectangular plates, composite laminated plates and shell structures under the action of low velocity 
impacts was investigated by Liu et al. in 1997 [4] who used nine-node finite elements. The histograms were drawn for the contact stress, displacement, and contact force. The researches found that the low velocity impact phenomena can be represented by using the finite element model. Iu et al. performed a study in 1998 [5] on the non linear vibration analysis of thin plates in presence of initial stress, by using spline finite strip method. The forced vibration has been studied under existence of initial stress and damping of the plate.

The deflection response of plate lied on an elastic foundation and subjected to an effect of a moving accelerated loads has been studied by Huang and Thambiratnam in 2001 [6] by using the finite strip method in analysis. This study refers to that when the load contacts the surface of plate then the structure behaves such as it is subjected to a suddenly applied load.

The dynamic behavior of orthotropic rectangular plates under the action of a moving force has been studied by Law et al. in 2003 [7]. The plate was supported on two parallel edges, the analysis was based on Lagrange equation. The authors studied the effects of some parameters on the dynamic response of the plates as vehicle velocity, dynamic amplification factor, plate length and the location of the moving force.

In the present study, a higher order finite layer with a second order polynomial has been used for the force vibration analysis of plates. Effects of many type of dynamic loads on the response of a rectangular plate has been studied, such as moving loads and impact loads.

\section{Finite layer method:}

The finite strip method pioneered in 1968 by Y.K. Cheung is an efficient tool for analyzing structures with regular geometric platform and simple boundary conditions. Basically, the finite strip method reduces a twodimensional problem to a onedimensional problem. By selecting functions satisfying the boundary conditions in two directions, the

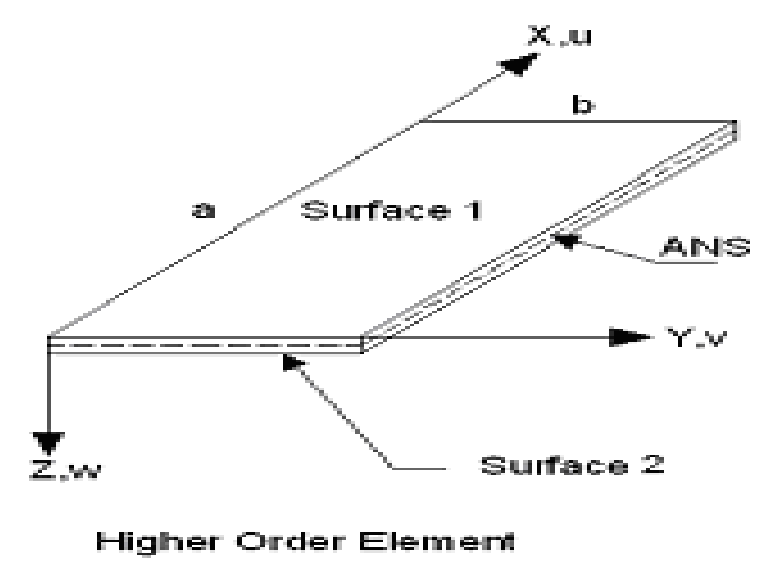


philosophy of the finite strip method can be extended to layer systems. The resulting method is called the finite layer method (FLM). The method was first proposed by Cheung and Chakrabarti (1971)[8]. The finite layer method is useful for layered materials, rectangular in planform. The higher order layer can be produced by introducing an auxiliary nodal surface at mid-distance between the upper and the lower surface of the lower order layer[9]. To illustrate the method, (Fig. 1) is considered.

(Fig. 1) Finite Layer Method

Let $\bar{z}=z / h$ then the lateral displacement component of the higher order layer, can be expressed as $[9,10]$ :

$\left.w=\sum_{m=1 n=1}^{r} \sum^{s} c 1 . w_{1 m n}+c 2 w_{2 m n}+c 3 . w_{3 m n}\right] X_{m}(x) Y_{n}(y)$

where

$$
c 1=2 \bar{z}^{2}-3 \bar{z}+1
$$

$c 2=-4 \bar{z}^{2}+4 \bar{z}$

$c 3=2 \bar{z}^{2}-\bar{z}$ .2

$w_{1 m n}, w_{2 m n}$ and $w_{3 m n}$ are displacement parameters for surface 1, auxiliary nodal surface and surface 2, respectively. The functions $X_{m}$ and $Y_{n}$ are taken as terms in a trigonometric series, satisfying the boundary conditions. In this manner a three-dimensional problem is reduced to onedimensional problem with considerable saving in computer storage and computational time [8].

By linear theory, the $(\mathrm{x}, \mathrm{y})$ displacement components $(\mathrm{u}, \mathrm{v})$ are linearly related to the derivatives of $w$; that is 
$u=A \frac{\partial w}{\partial x}, v=B \frac{\partial w}{\partial y}$

.........3

Thus, as with Eq. (1), then

$$
\begin{aligned}
& u=\sum_{m=1}^{r} \sum_{n=1}^{s}\left[c 1 \cdot u_{1 m n}+c 2 \cdot u_{2 m n}+c 3 \cdot u_{3 m n}\right] X_{m}(x) \cdot Y_{n}^{\prime}(y) \\
& v=\sum_{m=1 n=1}^{r} \sum_{n=1}^{s}\left[c 1 \cdot v_{1 m n}+c 2 \cdot v_{2 m n}+c 3 \cdot v_{3 m n}\right] X_{m}^{\prime}(x) \cdot Y_{n}(y)
\end{aligned}
$$

in which $X_{m}^{\prime}$ and $Y_{n}^{\prime}$ are the first derivatives of $X_{m}$ and $Y_{n}$ respectively. The displacement vector is

$$
\{f\}=\left\{\begin{array}{l}
u \\
v \\
w
\end{array}\right\}=\sum_{m=1}^{r} \sum_{n=1}^{s}[N]_{m n}\{\delta\}_{m n}=[N]\{\delta\}
$$

where

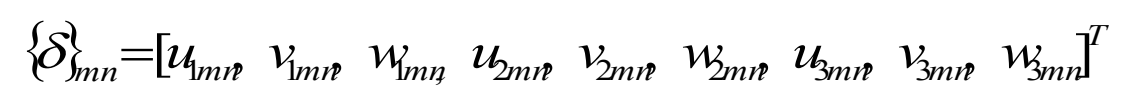

The strain-nodal displacement relationship is obtained as[11]:

$$
\{\varepsilon\}=\left\{\begin{array}{c}
\varepsilon_{x x} \\
\varepsilon_{y y} \\
\varepsilon_{z z} \\
\varepsilon_{x y} \\
\varepsilon_{y z} \\
\varepsilon_{z x}
\end{array}\right\}=\left\{\begin{array}{c}
\partial u / \partial x \\
\partial v / \partial y \\
\partial w / \partial z \\
\frac{1}{2}(\partial u \partial y+\partial v \partial x) \\
\frac{1}{2}(\partial v \partial z+\partial w \partial y) \\
\frac{1}{2}(\partial w \partial x+\partial u \partial z)
\end{array}\right\}=\sum_{m=1}^{r} \sum_{n=1}^{s}[B]_{m n}\{\delta\}_{m n}=[B]\{\delta\}
$$

The stress-strain relationship is 


$$
\{\sigma\}=\left[\begin{array}{llllll}
\sigma_{x x} & \sigma_{y y} & \sigma_{z z}, & \sigma_{x y} & \sigma_{y z} & \sigma_{z x}
\end{array}\right]^{T}=[D][B]\{\delta\}
$$

By applying the minimum total potential energy, the stiffness matrix can be derived in the following form

$$
[S]_{m n p q}=\int_{0}^{a} \int_{0}^{b} \int_{0}^{c}[B]_{m n}^{T}[D][B]_{p q} d x \cdot d y \cdot d z
$$

where $[S]_{m n p q}$ is the generalized stiffness matrix, which has the following expanded form:

$$
[S]=\left[\begin{array}{ccccccccc}
S_{1111} & S_{1112} & \ldots & S_{111 s} & S_{1121} & \ldots & S_{112 s} & \ldots & S_{11 r s} \\
S_{1211} & S_{1212} & \ldots & S_{121 s} & S_{1221} & \ldots & S_{122 s} & \ldots & S_{12 r s} \\
\ldots & & & & & & & & \\
S_{1 s 11} & S_{1 s 12} & & S_{1 s 1 s} & S_{1 s 21} & & S_{1 s 2 s} & & S_{1 s r s} \\
S_{2111} & S_{2112} & & S_{211 s} & S_{2121} & & S_{212 s} & & S_{21 r s} \\
\ldots & & & & & & & \\
S_{2 s 11} & S_{2 s 12} & & S_{2 s 1 s} & S_{2 s 21} & & S_{2 s 2 s} & & S_{2 s r s} \\
\ldots & & & & & & & \\
S_{r s 11} & S_{r s 12} & & S_{r s 1 s} & S_{r s 21} & & S_{r s 2 s} & & S_{r s r s}
\end{array}\right]
$$

\section{Consistent mass matrix of the layer:}

Studies have clarified that the consistent mass matrix is preferable in dynamic analysis problems because it is gives a more accurate results than the lumped mass matrix [12].

If the mass is distributed uniformly through each layer then each acceleration leads to generate a distributed inertia force which is [13] 
$\{q\}=-\rho \frac{d^{2}\{f\}}{d t^{2}}=-\rho[N] \frac{\partial^{2}\{\delta(t)\}}{\partial t^{2}}$

.11

where $\rho$ is the mass per unit volume, the general displacement $f$ depends on time.

Corresponding to natural vibrations, all points of a vibration system move in the same phase or in ordinary mode then $[14,15]$ :

$\{\delta(t)\}=\{\delta\} \cdot \sin (\omega t)$

..............12

Substituting Eq. (12) in Eq. (11), then

$$
\{q\}=-\rho[N] \cdot \omega^{2}\{\delta\} \cdot \sin (\omega t)
$$

By dropping $\sin (\omega t)$ from Eq. (13)[16], then

$$
\{q\}=-\rho[N] . \omega^{2}\{\delta\}
$$

By applying the principle of virtual work the nodal forces which are equivalent to any uniformly distributed load as $\{q\}$ can be found as follows[17]:

$$
\begin{aligned}
& \begin{aligned}
\{F\} & =\int_{v o l}[N]^{T} \cdot\{q\} \cdot d(\text { vol } .)=\int_{v o l} \rho \omega^{2}[N]^{T}[N]\{\delta\} d(\text { vol }) \\
& =\omega^{2}[M]\{\delta\}
\end{aligned} \\
& \therefore[M]_{\text {mnpq }}=\int_{\text {vol }} \rho \cdot[N]_{m n}^{T} \cdot[N]_{p q} \cdot d(\text { vol })
\end{aligned}
$$




\section{Forced vibration analysis of plates:}

When the plate is exposed to the dynamical loads then the plate movement can be expressed by a differential equation called forced vibration equation and has the following form

$$
[M] .\{\ddot{\delta}\}+[C] .\{\dot{\delta}\}+[S] .\{\delta\}=\{R\}
$$

where $[M],[C],[S]$ and $\{R\}$ are the consistent mass matrix, damping matrix, stiffness matrix and vector of external applied forces on the plate. The damping matrix can be found in a simplified manner as it is given by (Rayleigh), the suitable mathematical shape of calculating the damping matrix for a plate is as follows $[18,19]$

$[C]=\alpha \cdot[S]+\lambda \cdot[M]$

where

$$
\lambda=\frac{2 \cdot \xi \cdot \omega_{1} \cdot \omega_{2}}{\omega_{1}+\omega_{2}} \text {, and } \alpha=\frac{2 \cdot \xi}{\omega_{1}+\omega_{2}}
$$

and $(\xi)$ is the damping ratio and $\left(\omega_{1}\right.$, and $\left.\omega_{2}\right)$ are the first and the second natural frequencies of the plate and given in $(\mathrm{rad} / \mathrm{sec})$. Eq. (17) can be solved by various mathematical methods which may be explicit or implicit and each of them includes time integration manner [20]. The implicit integration manner is stable and unconditionally; the Newmark method is one of them and used in this study. Thus it can be express the relation between values of derivatives of vector $\{\delta\}$ at the time $(t+\Delta t)$ and the values at the time $(t)$ as:

$$
\begin{aligned}
& \{\ddot{\delta}\}_{t+\Delta t}=a_{0}\{\Delta \delta\}-a_{2}\{\dot{\delta}\}_{t}-a_{3}\{\ddot{\delta}\}_{t} \\
& \{\dot{\delta}\}_{t+\Delta t}=\{\dot{\delta}\}_{t}+a_{6}\{\ddot{\delta}\}_{t}+a_{7}\{\ddot{\delta}\}_{t+\Delta t}
\end{aligned}
$$


To guarantee the suitability and accuracy of the Newmark method the values of its parameters $(\beta$, and $\gamma)$ are taken as follows:

$\beta \geq 0.25(0.5+\gamma)^{2}$

$\gamma \geq 0.5$ .20

and the best value of these parameters are [21] $(\beta=0.25, \gamma=0.5)$. Values of variables ( $a_{0}$ to $\left.a_{7}\right)$ depend on $\beta$ and $\gamma$.

Two cases of applied loads are considered which are given as follows:

1- Moving force: It is a simple model of load which consists of a lonely single force moving on the surface of a plate. The force may represent a vehicle or any moving things that have a mass effecting on the plate and producing a dynamic vibration of it. If $\left(m_{v}\right)$ is the moving mass on plate and $(g)$ is the gravity acceleration then the moving force (weight) can be written as $[3,22]$

$p=m_{v} \cdot g$

Thus the force vector for a each layer is

$\{R\}=\{0,0, \quad p, 0,0,0,0,0,0\}^{T}$

2- Time dependent load: The plate may be exposed to time-varying loads as impact loads, explosions and seismic forces. These loads are represented by uniformly distributed loads or patch loads in this study. The used vector for dynamic load in the finite layer method is given as:

$$
\{R\}=\int[N]^{T} \cdot\{q\} \cdot d(\text { area })
$$

Where $(q)$ is the value of the uniformly distributed or patch load.

Steps for analyzing forced vibration: 
The forced vibration can be analyzed by finding the solution for the Eq. (17), by taking the value for $\{\Delta \delta\}$ as the change in value(ascending or descending) of the vector $\{\delta\}$ and this change occurs during the time step from $(t)$ to $(t+\Delta t)$. By using Newmark integration method then the relation can be expressed between the values of derivatives of vector $\{\delta\}$ at a time $(t+\Delta t)$ and the values at a time $(t)$ as follows:

$\{\ddot{\delta}\}_{t+\Delta t}=a_{0}\{\Delta \delta\}-a_{2}\{\dot{\delta}\}_{t}-a_{3}\{\ddot{\delta}\}_{t}$

$\{\dot{\delta}\}_{t+\Delta t}=\{\dot{\delta}\}_{t}+a_{6}\{\ddot{\delta}\}_{t}+a_{7}\{\ddot{\delta}\}_{t+\Delta t}$

..............24

by using the parameters ( $\beta$, and $\gamma$ ), the variables in Eq. (23) can be expressed as follows [23]:

$$
\begin{aligned}
& a_{0}=\frac{1}{\beta(\Delta t)^{2}} a_{1}=\frac{\gamma}{\beta \cdot t}, a_{2}=\frac{1}{\beta \cdot \Delta t}, a_{3}=\frac{1}{2 \beta}-1 \\
& a_{4}=\frac{\gamma}{\beta}-1, a_{5}=\frac{\Delta t}{2}\left(\frac{\gamma}{\beta}-2\right), a_{7}=\gamma \cdot \Delta t, a_{6}=\Delta t(1-\gamma)
\end{aligned}
$$

The values for initial velocity and displacement is assumed equal to zero thus the values for initial acceleration vectors at time $(t=0)$ are as follows:

$$
\{\ddot{\delta}\}_{0}=[M]^{-1}\left(\{R\}_{0}-[C]\{\dot{\delta}\}_{0}-[S]\{\delta\}_{0}\right)
$$

Then the applied stiffness matrix can be formulated as

$$
[\bar{S}]=[S]+a_{0}[M]+a_{1}[C]
$$

Thus it is possible at any time step to calculate the dynamic response in the following steps:

1- Calculate the applied force vector at time $(t+\Delta t)$ as follows: 


$$
\begin{aligned}
& {[\bar{R}]_{t+\Delta t}=[R]_{t+\Delta t}+[M]\left(a_{0}\{\delta\}_{t}+a_{2}\{\dot{\delta}\}_{t}+a_{3}\{\ddot{\delta}\}_{t}\right)+} \\
& {[C]\left(a_{1}\{\delta\}_{t}+a_{4}\{\dot{\delta}\}_{t}+a_{5}\{\ddot{\delta}\}_{t}\right)}
\end{aligned}
$$

2- Calculate the values of displacements at time $(t+\Delta t)$ as follows:

$$
\begin{aligned}
& {[\bar{S}]\{\delta\}_{t+\Delta t}=\{\bar{F}\}_{t+\Delta t}} \\
& \Rightarrow \quad\{\delta\}_{t+\Delta t}=[\bar{S}]^{-1}\{\bar{F}\}_{t+\Delta t}
\end{aligned}
$$

3- Calculate values of vectors of accelerations and velocities at time ( $t+\Delta t$ ) by Eq. (24).

In the problems which are related to plates excited by moving forces the location of the moving force on the surface of the plate is found at each time step by finding the $(\mathrm{x}, \mathrm{y})$ coordinates for applied point of force as follows:

$$
\begin{aligned}
& x_{t+\Delta t}=x_{t}+v_{t}^{x} \cdot \Delta t+\frac{1}{2} \cdot \dot{v}_{t}^{x} \cdot(\Delta t)^{2} \\
& y_{t+\Delta t}=y_{t}+v_{t}^{y} \cdot \Delta t+\frac{1}{2} \cdot \dot{v}_{t}^{y} \cdot(\Delta t)^{2}
\end{aligned}
$$

where $\left(x_{t+\Delta t}\right.$, and $\left.y_{t+\Delta t}\right)$ are the coordinates of the force contact location on the surface of the plate. $\left(v_{t}^{x}\right.$, and $\left.\dot{v}_{t}^{x}\right)$ represent the acceleration and the velocity of the moving force in $\mathrm{X}$-direction, $\left(v_{t}^{y}\right.$, and $\left.\dot{v}_{t}^{y}\right)$ represent the acceleration and the velocity in the Y-direction.

\section{Numerical applications:}

A- Analysis of plate excited by suddenly applied uniformly distributed load: The response of a simply supported square plate has been investigated, the material and geometrical properties of the plate are as follows : 
Plate length $(\mathrm{a})=10$ in $=0.254 \mathrm{~m}$

Plate thickness $(\mathrm{c})=0.5$ in $=0.0127 \mathrm{~m}$

Density $(\rho)=0.259 \times 10^{-3} \mathrm{lb} \cdot \mathrm{sec} / \mathrm{in}^{4}=4.8 \times 10^{-11} \mathrm{~kg} \cdot \mathrm{sec} / \mathrm{m}^{4}$

Modulus of elasticity $(\mathrm{E})=10 \times 10^{6} \mathrm{psi}=68947547.9 \mathrm{kN} / \mathrm{m}^{2}$

Poisson's ratio $(u)=0.3$

The uniformly distributed applied load is shown in Fig. (2). The higher order finite layer method is applied for analysis of the plate and the result is compared with that found by (Huang) [24] who used the rule of Gauss-Legendre $3 \times 3$ points in his analysis, the comparison is shown in Fig. (3). It is found that the finite layer results are in good agreement with the finite element results.

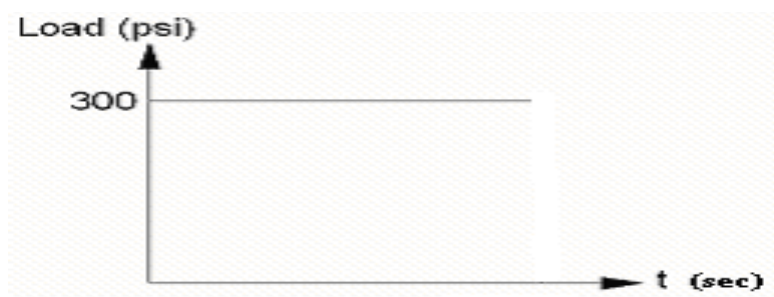

Fig. (2) Suddenly applied uniformly distributed load (Example-A) 


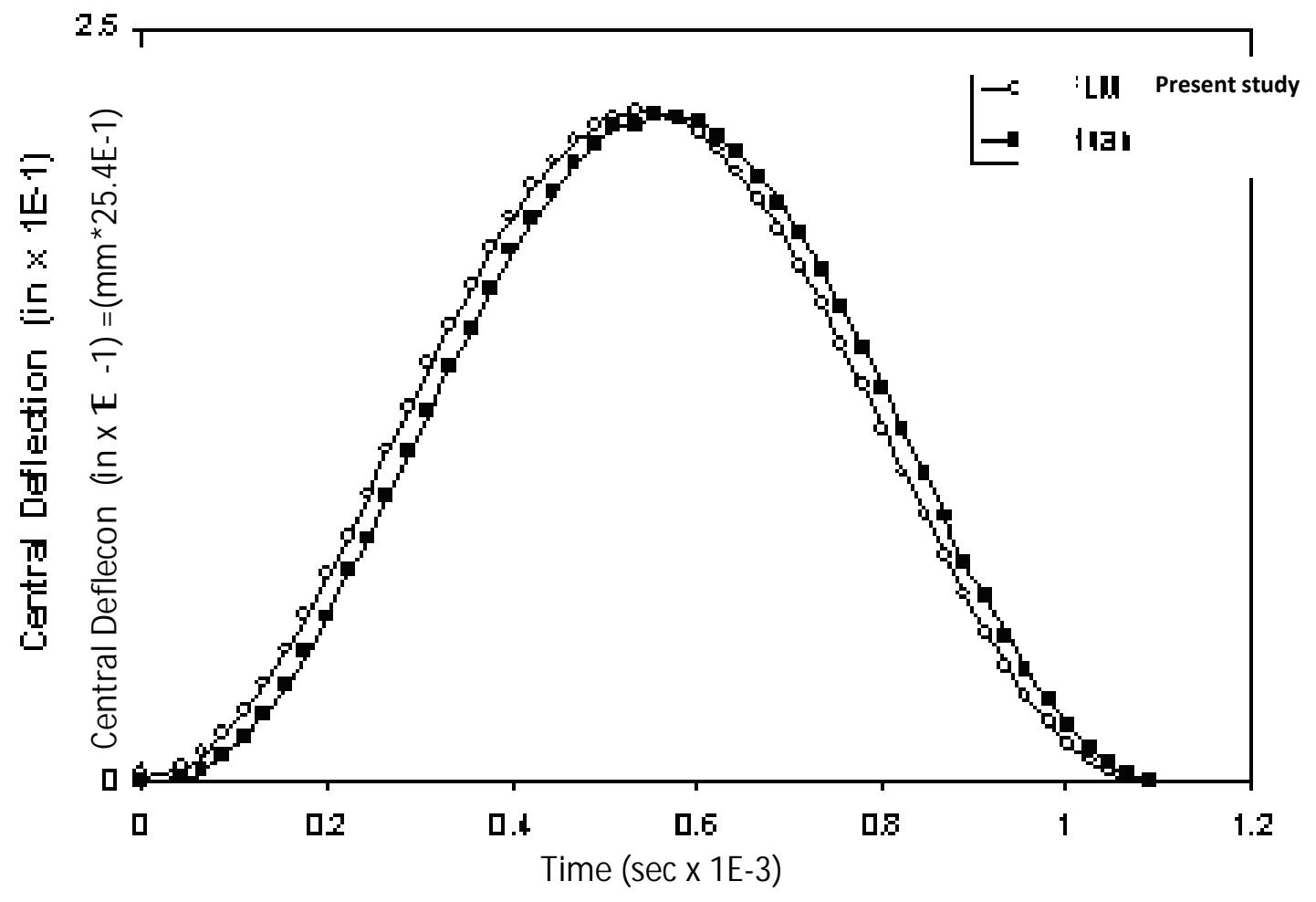

Fig. (3) Histogram of central response of plate (Example-A)

B- Analysis of plate excited by suddenly applied patch load: The plate shown in Fig. (4) is analyzed dynamically by the higher order finite layer method, the plate is subjected to a patch load of magnitude of $(\sqrt{2}$ psi) and the geometrical and material dimensionless properties are given in Fig. (4). The present results are compared with the previous results as given by (Huang) [24] in Fig. (5). It is found that the finite layer results are in good agreement with the results of reference[24].
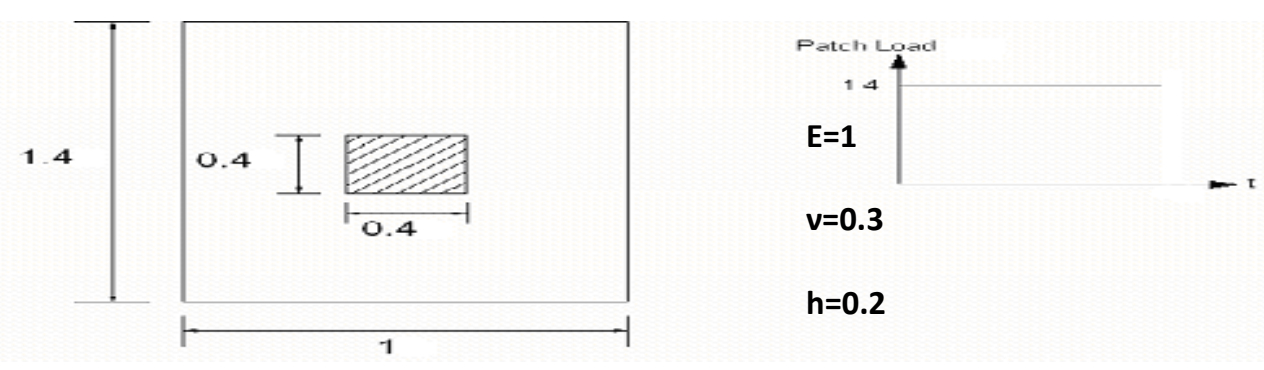

Fig. (4) Suddenly applied patch load (Example-B) 


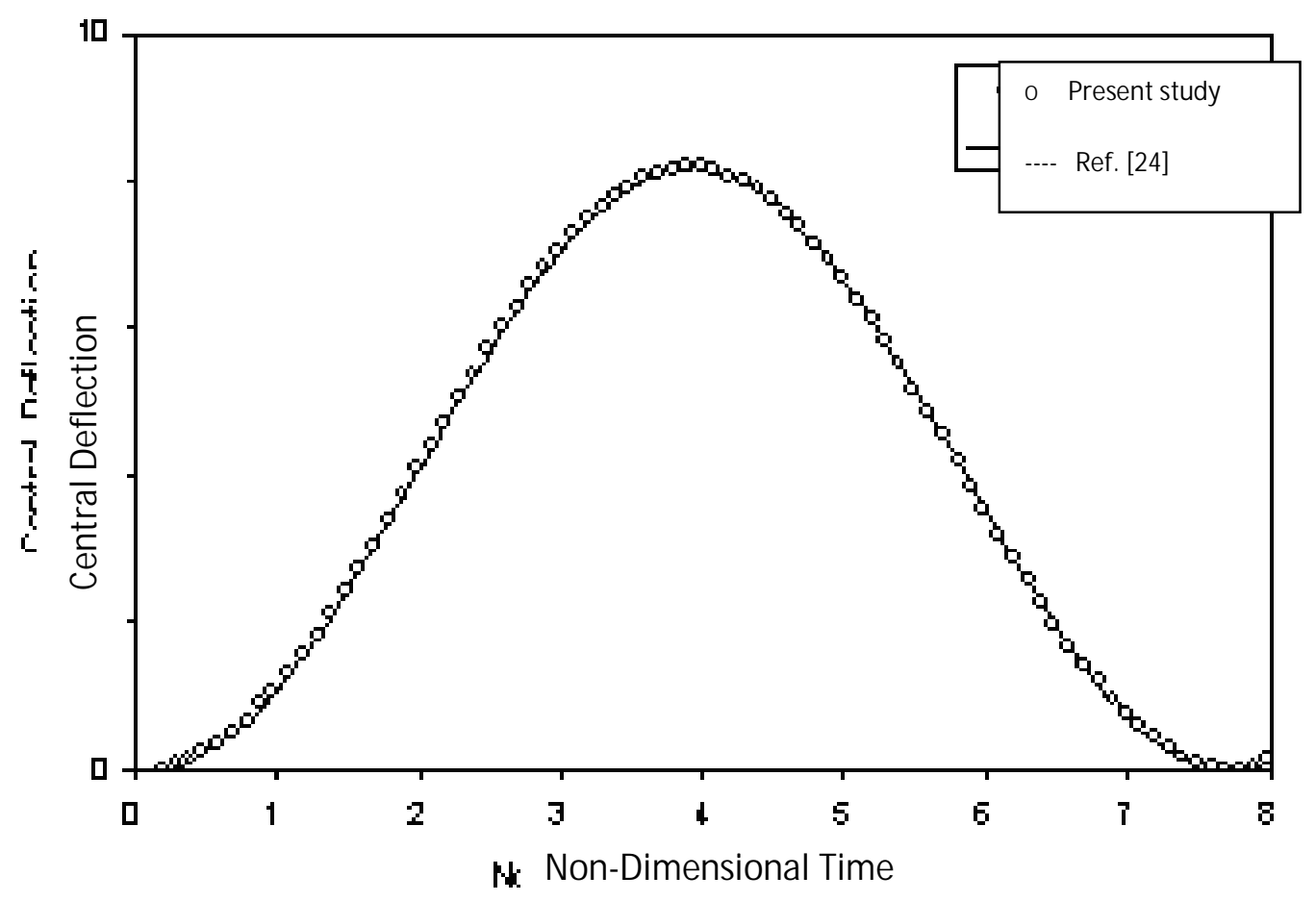

Fig. (5) Histogram of central response of plate (Example-B)

\section{C- Forced vibration analysis of plate subjected to a moving single} force: This example includes the analysis of undamped forced vibration of a square plate subjected to moving force $(2 \mathrm{lb})$. The force is moving with a velocity (234 mile/ hour (mph)) on the central line of the plate as in Fig. (6). The dimensions for the plate are given as follows:

$a=4$ in, $b=4$ in, $c=0.1$ in, $E=30 \times 10^{6}$ psi

$\rho=0.001 \quad l b \cdot \sec ^{2} /$ in $^{4}, \quad v=0.3$

Figs. (7) and (8) show the comparison of the results found by using (4) higher order layers with the results found by (Ting et al.) [3] who used the manner of structural impedance, the results are represented by dynamic magnification factor (DAF) and normalized displacements. 


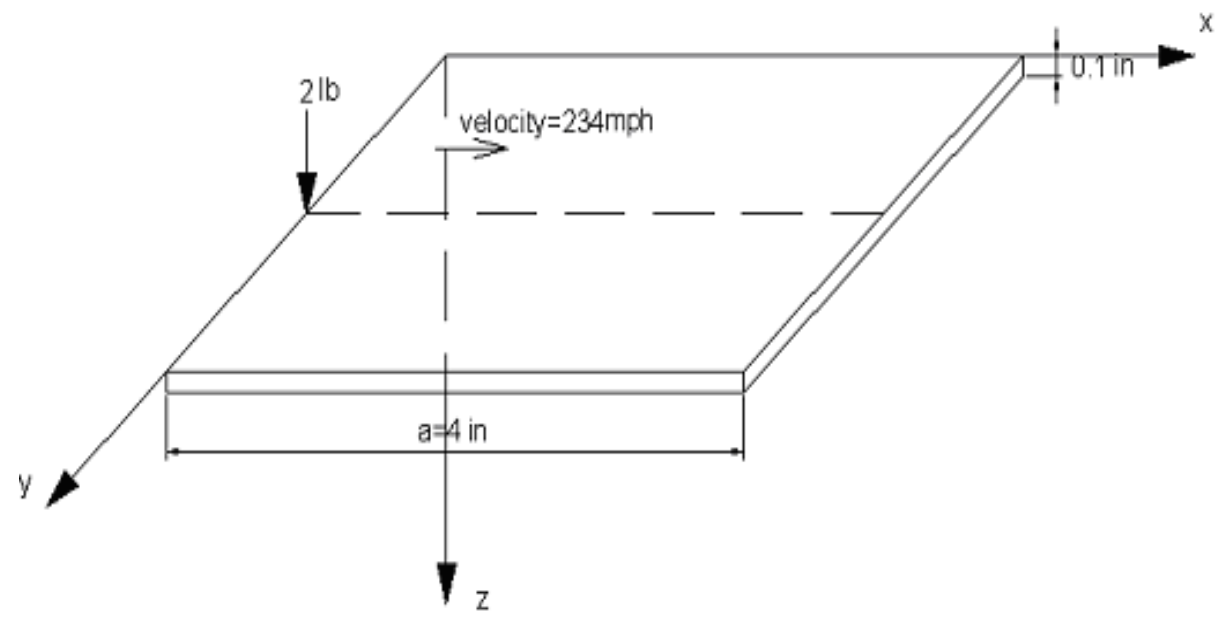

Fig. (6) Square plate subjected to a moving force

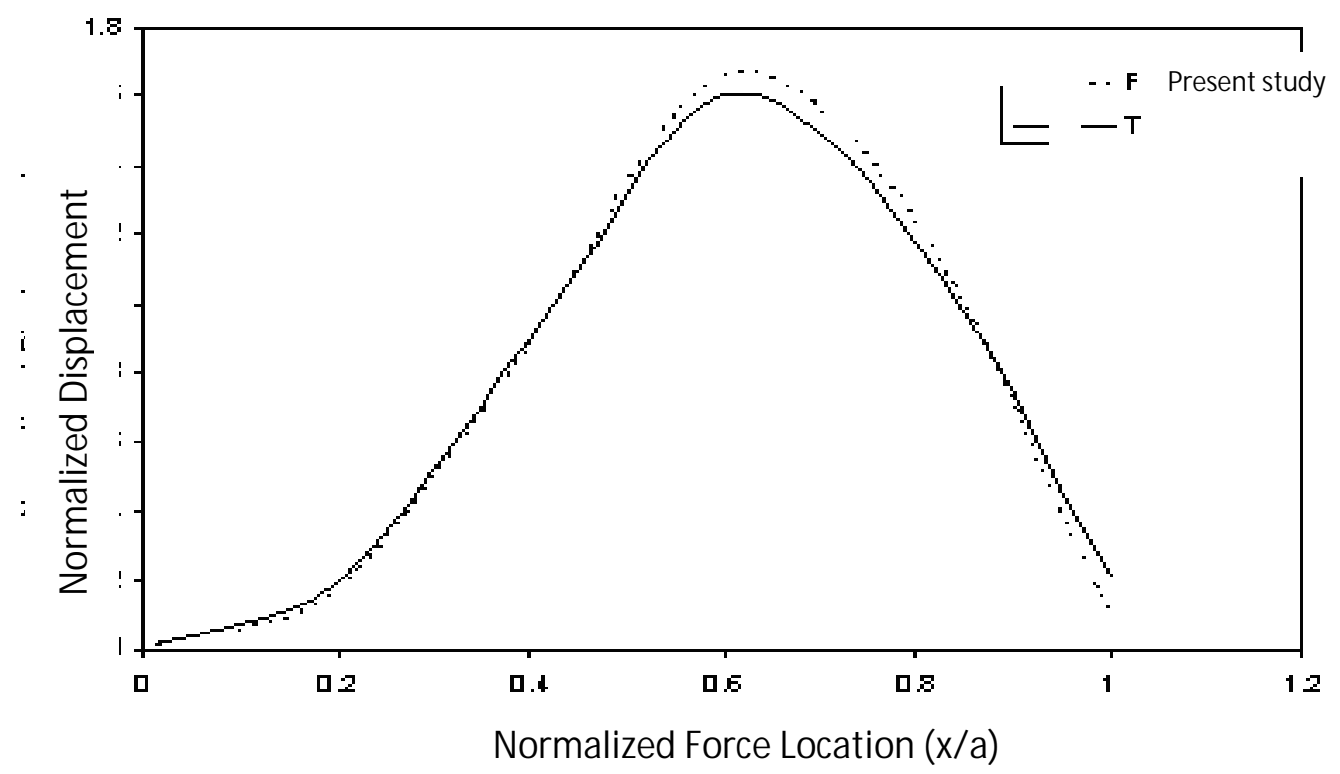

Fig. (7) Normalized displacements for a plate subjected to moving force 


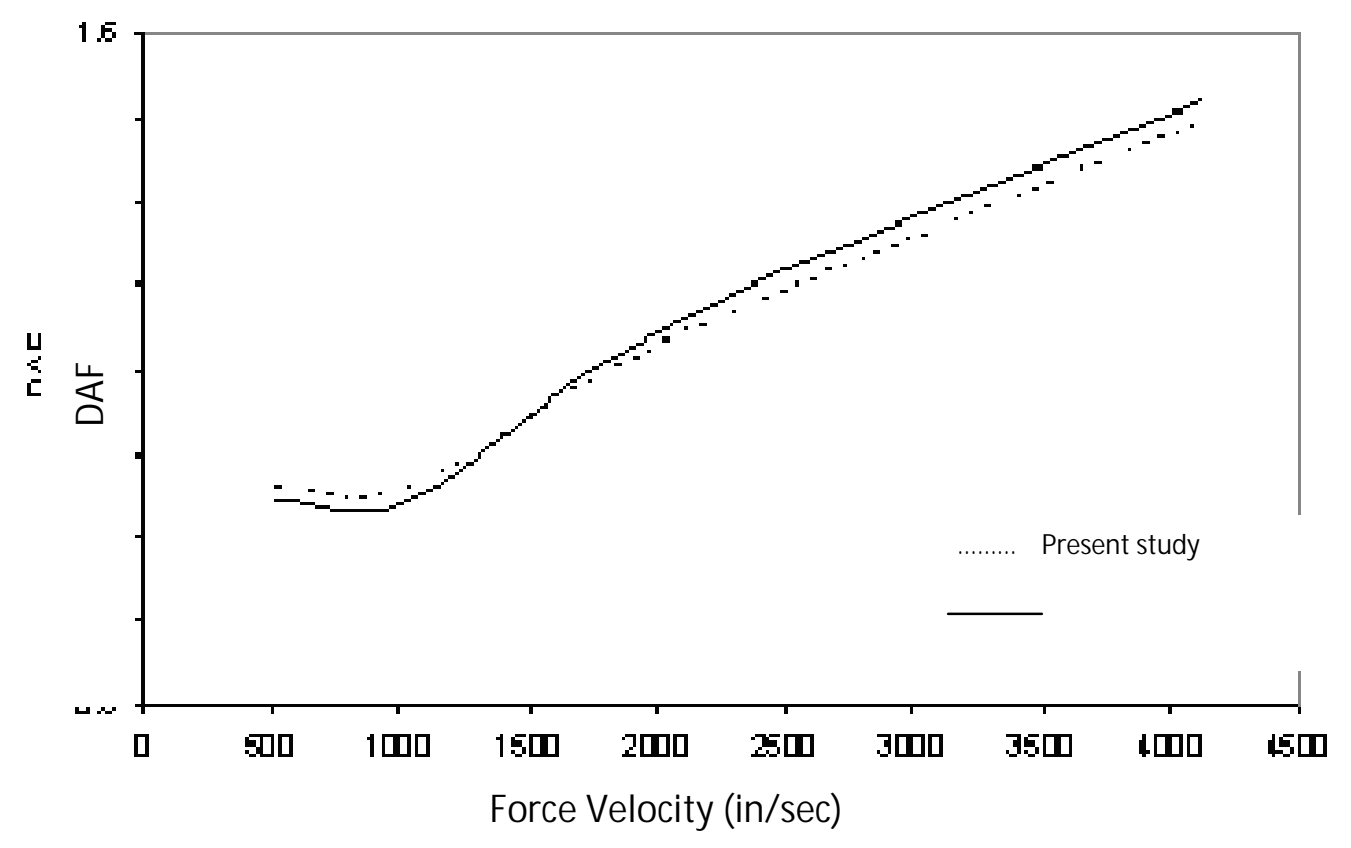

Fig. (8) Relation between dynamic amplification factor with force's velocity

D- Parametric study: The effects of damping of plate on the response of it has been considered in this example, this is done by considering the plate properties in example (C) but with using different damping ratios. The relations between the normalized displacements and the velocity of the moving force with the damping ratio have been found by making diagrams of Figs. (9-11).

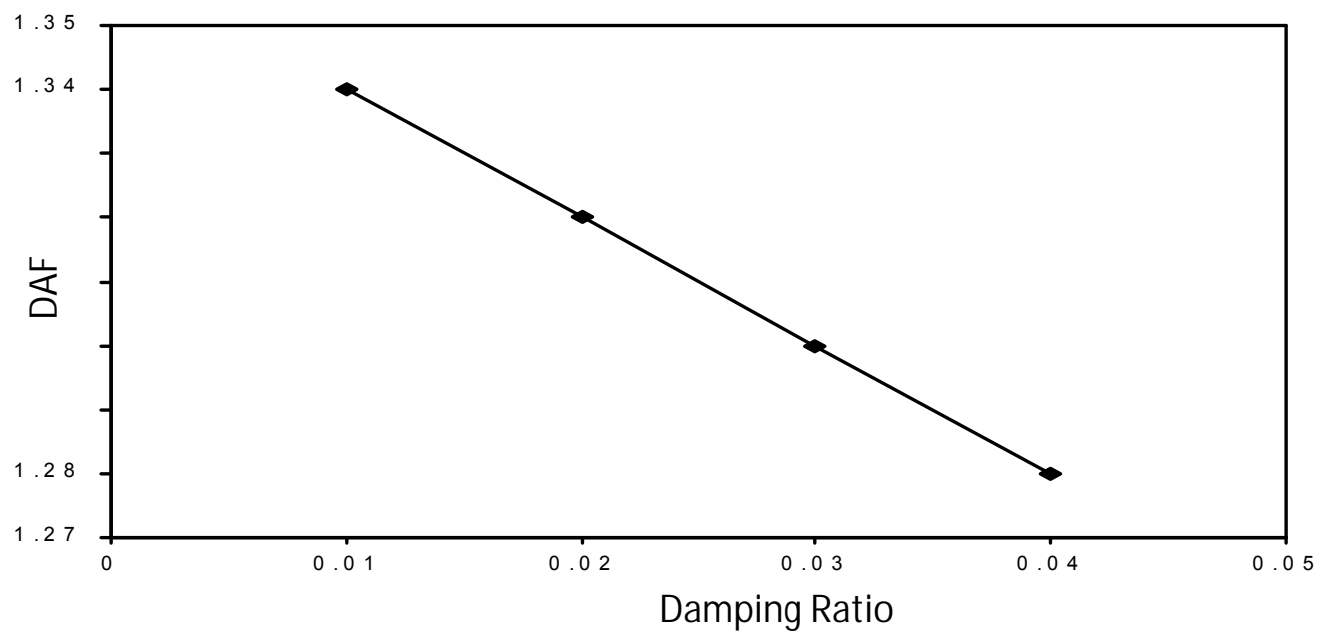


Fig. (9) Relation between DAF and damping ratio

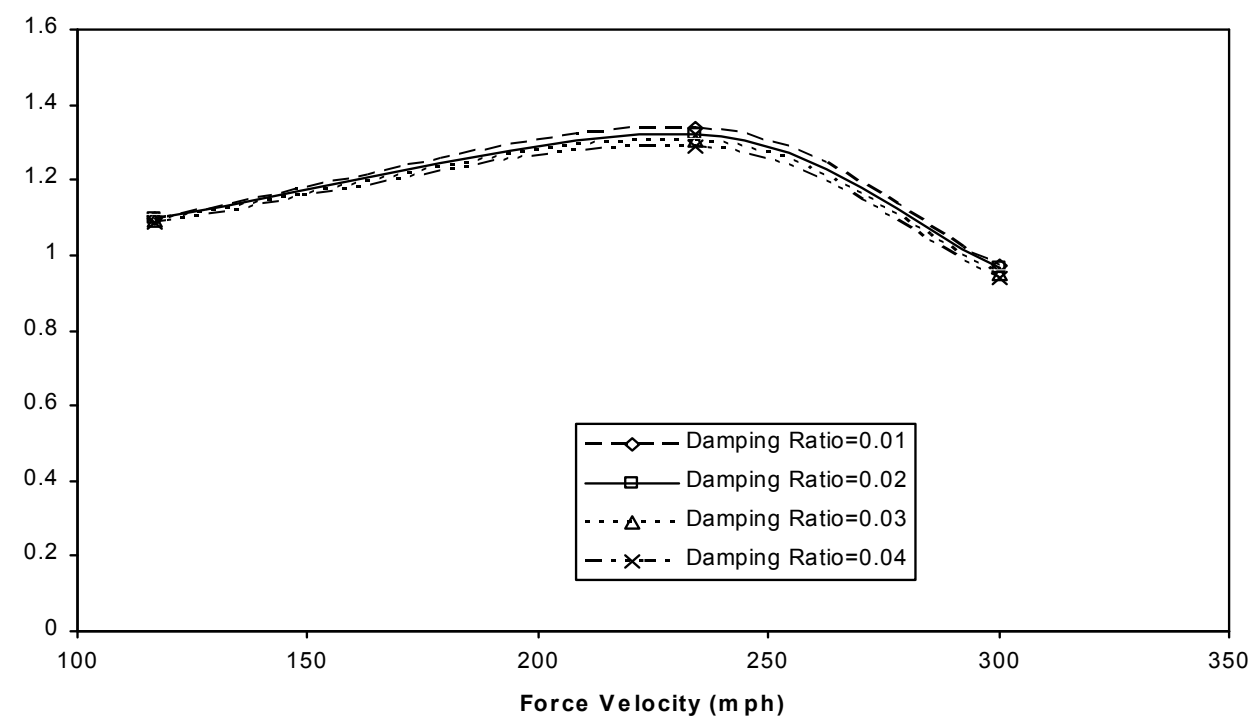

Fig. (10) Relation of moving force's velocity and DAF with damping ratio

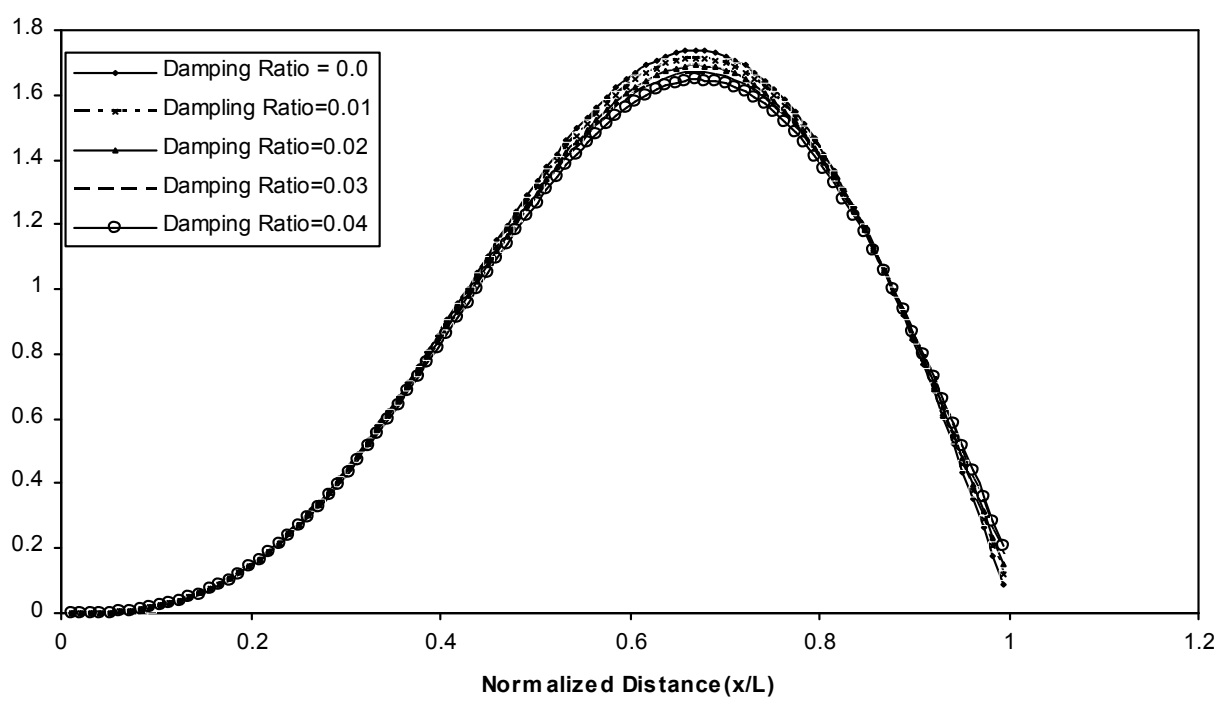

Fig. (11) Effect of damping on the normalized displacements of the plate 


\section{Conclusion:}

In the present study, the higher order finite layer method was used for dynamic analysis of simply supported plates that have a single span. According to this study the following points are concluded:

1- In the case of studying the vibration of a plate subjected to moving loads it is clear that by changing the value of the damping ratio of the plate the critical velocity remains constant, the critical velocity is the moving force velocity at which the magnitude of the dynamic amplification factor is a maximum.

2- The normalized displacement is increased by increasing the moving distance of the moving force on the surface of the plate until it reaches a specific limit (approximately (0.7) of the path of the moving force) after that it begins in decreasing.

3- By increasing the damping ratio of the plate, the normalized displacement will be decreased.

4- The dynamic amplification factor increased by increasing the velocity of the moving force until the velocity reach the limit of critical velocity then the curve of dynamic amplification factor will be descending.

5- The relation between the dynamic amplification factor and the damping ratio is in reverse relation. This shows that by increasing the damping ratio the dynamic amplification factor will be decreased and also the normalized displacement will be decreased.

\section{References:}

1. Chen H.L., Spyrakos C.C. and Venkatesh G. "Evaluating structural deterioration by dynamic response", Journal of Structural Engineering. Vol. 121, No. 8, Aug. 1995, pp. 1197-1204.

2. Whittaker W.L. and Christiano P. "Response of a plate and elastic half-space to harmonic waves", Earthquake Engineering and Structural Dynamics. Vol. 10, 1982, pp. 255-266.

3. Mechael R.T. and Ting E.C. "Dynamic response of the plate to moving loads; finite element method", Computers and Structures. Vol. 34, No. 3, 1990, pp. 509-521. 
4. Zishun Liu and Somsak Swaddiwudhipong "Response of the plate and shell structures due to velocity impact", Journal of engineering Mechanics. Vol. 123, No. 12, Dec. 1997, pp. 12301237.

5. Cheung Y.K. Zhu D.S. and Iu V.P. "Non-linear vibration of thin plates with initial stress by spline finite strip method", ThinWalled Structures. Vol. 32, 1998, pp. 275-287.

6. Huang M.H. and Thambiratnam "Deflection response of plate on Winkler foundation to moving accelerated loads", Engineering Structures. Vol. 23, 2001, pp. 1134-1141.

7. Zhu X.Q. and Law S.S. "Dynamic behavior of orthotropic rectangular plates under moving loads", Journal of Engineering Mechanics. Vol. 129, No. 1, Jan. 2003, pp. 79-87.

8. Cheung Y.K. and Tham L.G. (1998) The finite strip method, $\boldsymbol{C R C}$ Press, Boca Raton, $\boldsymbol{F L}$.

9. Majeed A.S. "Analysis of thick rectangular plates by the finite layer method", M.Sc. Thesis, Dept. of Civil Eng., University of Mosul, Mosul, 1999.

10.Haido J.H. "Dynamic analysis of rectangular plates using higher order finite layer", M.Sc. Thesis, Dept. of Civil Eng., University of Mosul, Mosul, 2006.

11.Boresi A.P. and Chong K.P. (2000) Elasticity in engineering mechanics, Wiley, New York.

12.Farfard M., Laflamme M., Savard M. and Bennur M. "Dynamic analysis of existing continuous bridge", ASCE, J. Bridge Engineering. Vol. 3, No.1, 1998, pp. 28-37.

13.Cook R.D., Malkus D.S. and Plesha M.E. (1989) Concepts and applications of finite element analysis", Wiley, New York.

14.Miquel C.J., Suarez B. and Onate E. "Dynamic analysis of structures using Reissner-Mindlin finite strip formulation", Computers and Structures. Vol. 31, No. 6, 1989, pp. 967-975.

15.Zhou D., Lo S.H., Au F.T.K. and Cheung Y.K. "Vibration analysis of rectangular Mindlin plates with internal line supports using static Timoshenko beam function", International Journal of Mechanical Science. Vol. 44, Nov. 2002, pp. 2503-2522.

16.Cheng Y.K. (1976) Finite strip method in structural analysis, Pergamon Press, Oxford.

17.Abdul-Razzak A.A. and Haido J.H. "Free Vibration o Rectangular Plates using Higher Order Finite Layer", AL-RAFIDAIN Engineering Journal. Vol. 15, 2007, pp. 19-32.

18.Smith L.M. and Griffiths D.V. (1998) Programming the finite element method, Third Edition. 
19.Tedescon J.W., Mcdougal W.G. and Ross C.A. (1999) Structural dynamic: Theory and Applications, Additional Wesley Longman, Inc. California.

20.Yang Y.B. and Yau J.D. "Vehicle-Bridge interaction element for dynamic analysis", Journal of Structural Engineering, ASCE. Vol. 123, No.11, 1997, pp. 1512-1518.

21.Yang Y.B., Yau J.D. and Hsu L.C. "Vibration of simple beams due to trains moving at high speed", Engineering Structures. Vol. 19, No. 11, 1997, pp. 936-944.

22.Green M.F. and Cebon D. "Dynamic interaction between heavy vehicles and highway bridges", Computers and Structures. Vol. 62, No. 2, 1997, pp. 253-264.

23.Wu Y.S. and Yang Y.B. "A versatile element for analyzing vehicle-bridge interaction response", Engineering Structures. Vol. 23, 2001, pp. 452-469.

24.Hou-Cheng Huang (1988) Static and dynamic analysis of plates and shells theory, software and applications. Swansea, UK. 